\section{Integral transforms solution for flow development in wavy wall ducts}

\section{Integral transforms solution}

219

Roseane L. Silva Mining Engineering Department, Universidade Federal do Pará, Marabá, Brazil

João N.N. Quaresma

School of Chemical Engineering, Universidade Federal do Pará, Belém, Brazil

Carlos A.C. Santos

Solar Energy Laboratory, Mechanical Engineering Department, Universidade Federal da Paraiba, João Pessoa, Brazil, and

Renato M. Cotta

Laboratory of Transmission and Technology of Heat,

Mechanical Engineering Department, Universidade Federal do Rio de Janeiro, Rio de Janeiro, Brazil

\begin{abstract}
Purpose - The purpose of this paper is to provide an analysis of two-dimensional laminar flow in the entrance region of wavy wall ducts as obtained from the solution of the steady Navier-Stokes equations for incompressible flow.

Design/methodology/approach - The study is undertaken by application of the generalized integral transform technique in the solution of the steady Navier-Stokes equations for incompressible flow. The streamfunction-only formulation is adopted, and a general filtering solution that adapts to the irregular contour is proposed to enhance the convergence behavior of the eigenfunction expansion.

Findings - A few representative cases are considered more closely in order to report some numerical results illustrating the eigenfunction expansions convergence behavior. The product friction factor-Reynolds number is also computed and compared against results from discrete methods available in the literature for different Reynolds numbers and amplitudes of the wavy channel.

Research limitations/implications - The proposed methodology is fairly general in the analysis of different channel profiles, though the reported results are limited to the wavy channel configuration. Future work should also extend the analysis to geometries represented in the cylindrical coordinates with longitudinally variable radius.

Practical implications - The error-controlled converged results provide reliable benchmark results for the validation of numerical results from computational codes that address the solution of the Navier-Stokes equations in irregular geometries.

Originality/value - Although the hybrid methodology is already known in the literature, the results here presented are original and further challenges application of the integral transform method in the solution of the Navier-Stokes equations.

Keywords Transforms, Laminar flow, Waves

Paper type Research paper
\end{abstract}

Received 28 September 2009

Revised 16 January 2010 Accepted 17 February 2010 


\section{HFF}

21,2

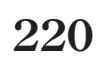

\section{Nomenclature}

$\begin{array}{llll}\mathrm{a}^{*} & =\text { Amplitude of the wavy boundary } & \mathrm{y} & =\text { Dimensionless transversal } \\ \mathrm{b} & =\text { Half the distance between the walls } & & \text { coordinate } \\ & \text { at the duct inlet } & \mathrm{y}_{1}, \mathrm{y}_{2} & =\text { Boundaries geometric profiles } \\ \mathrm{c} & =\text { Parameter of scale compression } & \mathrm{Y}_{\mathrm{i}} & =\text { Eigenfunctions }\end{array}$

$\mathrm{F}(\mathrm{x}, \mathrm{y})=$ Filtering function

$\mathrm{k}_{1}, \mathrm{k}_{2}=$ Streamfunction values at the duct walls

n $\quad=$ Unit normal vector

$\mathrm{N}_{\mathrm{i}} \quad=$ Norm

NTV $=$ Expansion truncation order

$\mathrm{p}=$ Dimensionless pressure field

$\mathrm{Q}=$ Dimensionless mass flux

Re $\quad=$ Reynolds number

$\mathrm{u} \quad=$ Dimensionless longitudinal velocity component

$\mathrm{v}=$ Dimensionless transversal velocity component

$\mathrm{x}=$ Dimensionless longitudinal coordinate

$\mathrm{x}_{\text {out }}=$ Value of the longitudinal coordinate at the duct outlet

\section{Greek letters}

$\alpha=$ Dimensionless duct amplitude

$\beta_{\mathrm{i}}=\mathrm{x}$-independent eigenvalues

$\eta \quad=$ Transformed longitudinal coordinate

$\xi=$ Transformed transversal coordinate

$\tau=$ Compressed longitudinal coordinate

$\phi \quad=$ Filtered potential

$\bar{\phi}_{i} \quad=$ Transformed potentials

$\psi \quad=$ Streamfunction

$\omega \quad=$ Vorticity

Subscripts and superscripts

$=$ Integral transformed quantities

$\overline{\mathrm{i}} \mathrm{j}, \mathrm{k}=$ Expansions indices

\section{Introduction}

Fluid flow within irregularly shaped ducts is found in several industrial applications related, for instance, to the flow of liquids in chemical processing plants; air flow in cooling, heating and ventilation units; and cooling of electronic equipment. In these applications, there is the need of evaluating certain physical parameters for proper thermal-hydraulic design, such as friction factors and heat-transfer coefficients. Channels with corrugated surfaces are, for example, employed in compact heat exchangers (Kays and London, 1984) for heat-transfer enhancement. Most theoretical studies performed on the fluid dynamics and thermal phenomena occurring in corrugated wall ducts consider corrugations having a periodical pattern which are described by simple functions such as rectangular, triangular or sinusoidal relations. A few experimental and theoretical studies are available in the literature on the thermohydraulics of such wavy wall ducts (Goldstein and Sparrow, 1977; Asako et al., 1988; Sunden and Trollheden, 1989; Xiao et al., 1989; Wang and Chen, 2002). Wall corrugation may also be employed aimed at promoting early transition of laminar to turbulent flows, sometimes responsible for the enhancement of heat transfer in practical applications (Cabal et al., 2001; Balaras, 2004; Dalal and Das, 2007; Marchioli et al., 2007). More recently, a few works have addressed the interest in investigating channel corrugations at the micro-scale, either for liquid or gaseous flows (Vasudevaiah and Balamurugan, 2001; Chen and Cho, 2007; Castellões and Cotta, 2008).

The numerical simulation of flows in irregularly shaped channels by the conventional discrete approaches requires sufficiently fine meshes and considerable computational effort so as to capture the detailed aspects of the fluid flow that influence the wall friction. On the other hand, a number of hybrid numerical-analytical approaches were progressively developed and presented in the open literature, 
advocated as less expensive and at least as companion tools for the more popular and, in general, more straightforward discrete numerical methods. Eigenfunction expansion-type approaches were among such extended analytical tools that to a considerable extent were also able to profit from the concurrent development of symbolic computation platforms (Wolfram, 1999). Within this class of approaches, the integral transform method was gradually expanded in its applicability, under the label of the generalized integral transform technique (GITT) (Cotta, 1993, 1994, 1998; Cotta and Mikhailov, 1997, 2006; Santos et al., 2001; Cotta et al., 2005), and extensively employed in heat/mass transfer and fluid flow problems. For instance, under either the boundary-layer or full Navier-Stokes formulations, a number of contributions have advanced this method towards the error-controlled solution of internal flow and convective heat-transfer problems (Pérez Guerrero and Cotta, 1992, 1995, 1996; Carvalho et al., 1993; Machado and Cotta, 1995; Figueira da Silva and Cotta, 1996, 1998; Lima et al., 1997, 2007; Quaresma and Cotta, 1997; Cotta and Pimentel, 1998; Pereira et al., 1998; Machado and Cotta, 1999; Pérez Guerrero et al., 2000; Naveira et al., 2007; $\mathrm{Paz}$ et al., 2007). Both the primitive variables and streamfunction (or vector-scalar potentials for three-dimensional flows) formulations were adopted in such developments, with some preference to the streamfunction form, due to the elimination of the pressure field and automatic satisfaction of the continuity equation. In the case of the streamfunction-only formulation, the appropriate eigenfunction expansion for the velocity problem is in general proposed based on a fourth-order eigenvalue problem related to the analytical solution of the linear biharmonic equation for vanishing Reynolds number. In the context of computational solutions with automatically controlled accuracy, the GITT (Wolfram, 1999; Cotta, 1993, 1994; Cotta and Mikhailov, 1997; Cotta, 1998; Santos et al., 2001; Cotta et al., 2005; Cotta and Mikhailov, 2006), with its automatic global error control capability, appears as a reliable path for obtaining benchmark results, allowing for a more definitive critical evaluation of previously published numerical results of classical test problems. The GITT has already been utilized to find hybrid analyticalnumerical solutions for laminar flow development inside parallel-plates channels (Carvalho et al., 1993; Machado and Cotta, 1995; Pérez Guerrero and Cotta, 1995; Figueira da Silva and Cotta, 1996; Lima et al., 2007), by using both the primitive variables and streamfunction-only formulations, in either the Navier-Stokes or boundary-layer formulations. Extending such efforts, the present work is motivated by the application of the GITT in the solution of hydrodynamic developing flow in a wavy wall duct. Thus, a Navier-Stokes-based formulation for two-dimensional laminar incompressible flow in irregularly shaped channels is adopted, in terms of the streamfunction only such as that one originally proposed and solved by integral transforms in (Pérez Guerrero et al., 2000). Here, the streamfunction is split up in two parts, where one of them represents a generic filtering solution, which adapts to the irregular boundary of the duct. The aim of the filtering solution is to offer a convergence enhancement effect on the eigenfunction expansion for the streamfunction, by utilizing an analytical filter that changes along the flow development. A wavy wall duct is then more closely studied and computations for the streamfunction, vorticity and velocity fields are performed, as well as for the product of the friction factor-Reynolds number, for different values of the governing parameters of the flow, such as the Reynolds number and the amplitude of the wavy surface, extending the scope and preliminary assessment of this problem as first presented in (Silva et al., 2007). Finally, a set of reference results are systematically presented and employed 
$\mathrm{HFF}$

21,2

222 to covalidate previously reported results (Wang and Chen, 2002) obtained by discrete numerical methods.

\section{Problem formulation and solution methodology}

We consider two-dimensional steady laminar flow of an incompressible Newtonian fluid in the inlet region of a duct of irregular geometry. Figure 1 shows the schematic representation of the considered internal flow problem, which is not required to be symmetrical with respect to the longitudinal axis in the proposed approach and associated algorithm. The flow is governed by the continuity and Navier-Stokes equations, and the following dimensionless variables are here employed:

$$
\begin{aligned}
& \mathrm{x}=\frac{\mathrm{x}^{*}}{\mathrm{~b}} ; \quad \mathrm{y}=\frac{\mathrm{y}^{*}}{\mathrm{~b}} ; \quad \mathrm{y}_{1}(\mathrm{x})=\frac{\mathrm{y}_{1}^{*}\left(\mathrm{x}^{*}\right)}{\mathrm{b}} ; \quad \mathrm{y}_{2}(\mathrm{x})=\frac{\mathrm{y}_{2}^{*}\left(\mathrm{x}^{*}\right)}{\mathrm{b}} ; \\
& \mathrm{u}=\frac{\mathrm{u}^{*}}{\mathrm{u}_{0}} ; \quad \mathrm{v}=\frac{\mathrm{v}^{*}}{\mathrm{u}_{0}} ; \quad \mathrm{p}=\frac{\mathrm{p}^{*}}{\rho \mathrm{u}_{0}^{2}} ; \quad \operatorname{Re}=\frac{\mathrm{bu}_{0}}{\nu}
\end{aligned}
$$

where $b$ represents half the distance between the walls at the duct inlet.

Adopting the streamfunction-only formulation (Pérez Guerrero et al., 2000), the problem is then written in dimensionless form as:

$$
\mathrm{L}_{1}[\psi, \psi]=\mathrm{L}_{2}[\psi]
$$

the operators $\mathrm{L}_{1}[\mathrm{f}, \mathrm{g}]$ and $\mathrm{L}_{2}[\mathrm{f}]$ are defined as:

$$
\begin{gathered}
L_{1}[f, g]=\frac{\partial f}{\partial y}\left(\frac{\partial^{3} g}{\partial x^{3}}+\frac{\partial^{3} g}{\partial x \partial y^{2}}\right)-\frac{\partial f}{\partial x}\left(\frac{\partial^{3} g}{\partial x^{2} \partial y}+\frac{\partial^{3} g}{\partial y^{3}}\right) \\
L_{2}[f]=\frac{1}{\operatorname{Re}}\left(\frac{\partial^{4} f}{\partial x^{4}}+2 \frac{\partial^{4} f}{\partial x^{2} \partial y^{2}}+\frac{\partial^{4} f}{\partial y^{4}}\right)
\end{gathered}
$$

and the boundary conditions are associated with no-slip and impermeability at the duct walls:

$$
\begin{gathered}
\psi\left(\mathrm{x},-\mathrm{y}_{1}(\mathrm{x})\right)=\mathrm{k}_{1} ; \quad \frac{\partial \psi\left(\mathrm{x},-\mathrm{y}_{1}(\mathrm{x})\right)}{\partial \mathbf{n}}=0 \\
\psi\left(\mathrm{x}, \mathrm{y}_{2}(\mathrm{x})\right)=\mathrm{k}_{2} ; \quad \frac{\partial \psi\left(\mathrm{x}, \mathrm{y}_{2}(\mathrm{x})\right)}{\partial \mathbf{n}}=0
\end{gathered}
$$

Figure 1.

Definition of the general irregular geometry for the problem and coordinates system

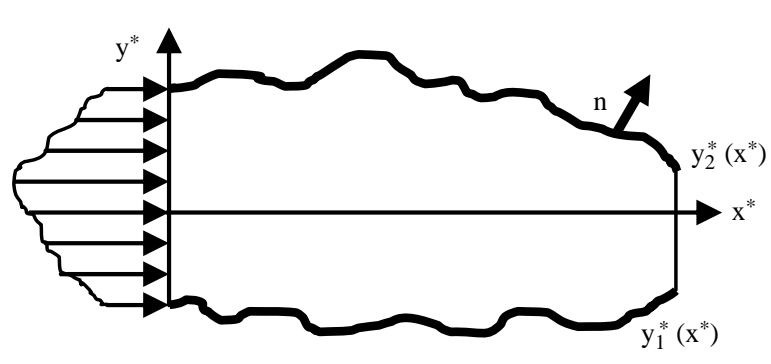


where $\mathbf{n}, \mathrm{k}_{1}$ and $\mathrm{k}_{2}$ represent, respectively, the unit normal vector in the outward direction of the duct wall and the streamfunction values at the walls. The constant $\mathrm{Q}$ represents the volumetric flow rate per unit of length and is determined as (Pérez Guerrero et al., 2000):

$$
\psi\left(0, \mathrm{y}_{2}\right)=\mathrm{k}_{2}=\mathrm{Q}+\mathrm{k}_{1}
$$

In the above equations, the definition of streamfunction was employed according to:

$$
\mathrm{u}(\mathrm{x}, \mathrm{y})=\frac{\partial \psi(\mathrm{x}, \mathrm{y})}{\partial \mathrm{y}}, \quad \mathrm{v}(\mathrm{x}, \mathrm{y})=-\frac{\partial \psi(\mathrm{x}, \mathrm{y})}{\partial \mathrm{x}}
$$

These definitions allow automatic satisfaction of the continuity equation and eliminate the pressure field from the problem formulation (equation (2a)).

In the solution of equation (2a) by using the GITT approach, it is convenient to define a filter in order to homogenize the boundary conditions in the y direction, which later will be the coordinate chosen for construction of the eigenvalue problem. Therefore, the general filtering function is defined from:

$$
\psi(\mathrm{x}, \mathrm{y})=\phi(\mathrm{x}, \mathrm{y})+\mathrm{F}(\mathrm{x}, \mathrm{y})
$$

where $\phi(\mathrm{x}, \mathrm{y})$ represents the unknown potential to be determined, and $\mathrm{F}(\mathrm{x}, \mathrm{y})$ is the filter, which at this point is only required to have the same values of $\psi(\mathrm{x}, \mathrm{y})$ at the duct walls. The function $\mathrm{F}(\mathrm{x}, \mathrm{y})$ is thus not necessarily a particular solution of $\psi(\mathrm{x}, \mathrm{y})$. Therefore, introducing equation (7) into equation (2a), results:

$$
\mathrm{L}_{1}[\phi, \phi]+\mathrm{L}_{1}[\phi, \mathrm{F}]+\mathrm{L}_{1}[\mathrm{~F}, \phi]+\mathrm{L}_{1}[\mathrm{~F}, \mathrm{~F}]=\mathrm{L}_{2}[\phi]+\mathrm{L}_{2}[\mathrm{~F}]
$$

with the filtered boundary conditions:

$$
\begin{gathered}
\phi\left(\mathrm{x},-\mathrm{y}_{1}\right)=\mathrm{k}_{1}-\mathrm{F}\left(\mathrm{x},-\mathrm{y}_{1}\right) ; \quad \frac{\partial \phi\left(\mathrm{x},-\mathrm{y}_{1}\right)}{\partial \mathbf{n}}=0 \\
\phi\left(\mathrm{x}, \mathrm{y}_{2}\right)=\mathrm{k}_{2}-\mathrm{F}\left(\mathrm{x}, \mathrm{y}_{2}\right) ; \quad \frac{\partial \phi\left(\mathrm{x}, \mathrm{y}_{2}\right)}{\partial \mathbf{n}}=0
\end{gathered}
$$

The filtering function can be built, for instance, by constructing at any cross-section along the duct a fully developed velocity profile, which follows the irregularity of the duct. In order to more easily obtain this filter, a relationship between the original coordinates system $(y, x)$ and a new transformed system $(\eta, x)$ is given as:

$$
\eta=\mathrm{y}-\mathrm{y}_{3}(\mathrm{x}) ; \quad \mathrm{y}_{0}(\mathrm{x})=\frac{1}{2}\left[\mathrm{y}_{1}(\mathrm{x})+\mathrm{y}_{2}(\mathrm{x})\right] ; \quad \mathrm{y}_{3}(\mathrm{x})=\frac{1}{2}\left[\mathrm{y}_{2}(\mathrm{x})-\mathrm{y}_{1}(\mathrm{x})\right]
$$

or in terms of the original coordinates:

$$
\mathrm{F}(\mathrm{x}, \mathrm{y})=\frac{3}{4} \mathrm{Q}\left[\left(\frac{\mathrm{y}-\mathrm{y}_{3}}{\mathrm{y}_{0}}\right)-\frac{1}{3}\left(\frac{\mathrm{y}-\mathrm{y}_{3}}{\mathrm{y}_{0}}\right)^{3}\right]+\frac{\mathrm{Q}}{2}+\mathrm{k}_{1}
$$

where $\mathrm{y}_{3}$ represents the distance between the axes $\mathrm{y}$ and $\eta$, while $\mathrm{y}$ belongs to the interval $\left[-\mathrm{y}_{1}(\mathrm{x}), \mathrm{y}_{2}(\mathrm{x})\right]$ and $\eta \in\left[-\mathrm{y}_{0}(\mathrm{x}), \mathrm{y}_{0}(\mathrm{x})\right]$. On the other hand, a fixed domain permits a more straightforward visualization of both this filtering solution and the 
$\mathrm{HFF}$

21,2

\section{4}

eigenvalue problem to be proposed, in terms of a new transversal coordinate, $\xi$. Therefore, the domain $\xi \in[-1,1]$ is defined from:

$$
\xi=\frac{\eta}{\mathrm{y}_{0}}=\frac{\mathrm{y}-\mathrm{y}_{3}}{\mathrm{y}_{0}}
$$

Thus, the filter can be rewritten in the form:

$$
\mathrm{F}(\xi)=\frac{3}{4}\left[\xi-\frac{\xi^{3}}{3}\right]+\frac{\mathrm{Q}}{2}+\mathrm{k}_{1}
$$

Now, in light of the homogeneous characteristics of the boundary conditions in the transversal direction, it is more appropriate to choose this coordinate for proposing the eigenfunction expansion, to be employed in the process of integral transformation. By considering the relation given by equation (13), the auxiliary fourth-order eigenvalue problem is taken as:

$$
\begin{gathered}
\frac{\mathrm{d}^{4} \mathrm{Y}_{\mathrm{i}}(\xi)}{\mathrm{d} \xi^{4}}=\left(\mu_{\mathrm{i}} \mathrm{y}_{0}\right)^{4} \mathrm{Y}_{\mathrm{i}}(\xi) \equiv \beta_{\mathrm{i}}^{4} \mathrm{Y}_{\mathrm{i}}(\xi) \\
\mathrm{Y}_{\mathrm{i}}(-1)=0 ; \quad \frac{\mathrm{d} \mathrm{Y}_{\mathrm{i}}(-1)}{\mathrm{d} \xi}=0 ; \quad \mathrm{Y}_{\mathrm{i}}(1)=0 ; \quad \frac{\mathrm{d} \mathrm{Y}_{\mathrm{i}}(1)}{\mathrm{d} \xi}=0
\end{gathered}
$$

Problem (15) is analytically solved, to furnish:

$$
\mathrm{Y}_{\mathrm{i}}(\xi)= \begin{cases}\frac{\cos \left(\beta_{\mathrm{i}} \xi\right)}{\cos \left(\beta_{\mathrm{i}}\right)}-\frac{\cosh \left(\beta_{\mathrm{i}} \xi\right)}{\cosh \left(\beta_{\mathrm{i}}\right)}, & i=1,3,5, \ldots \\ \frac{\sin \left(\beta_{\mathrm{i}} \xi\right)}{\sin \left(\beta_{\mathrm{i}}\right)}-\frac{\sinh \left(\beta_{\mathrm{i}} \xi\right)}{\sinh \left(\beta_{\mathrm{i}}\right)}, & i=2,4,6, \ldots\end{cases}
$$

where the $\mathrm{x}$-independent eigenvalue $\beta_{\mathrm{i}}$ is defined as, $\beta_{\mathrm{i}}=\mu_{\mathrm{i}}(\mathrm{x}) \mathrm{y}_{0}(\mathrm{x})$, and computed from the transcendental equations as follows:

$$
\tanh \left(\beta_{i}\right)=\left\{\begin{array}{cc}
-\tan \left(\beta_{i}\right), & i=1,3,5, \ldots \\
\tan \left(\beta_{i}\right), & i=2,4,6, \ldots
\end{array}\right.
$$

Also, the eigenfunctions satisfy the following orthogonality property:

$$
\int_{-y_{1}}^{y_{2}} Y_{i} Y_{j} d y= \begin{cases}0, & \text { for } i \neq j \\ N_{i}(x)=2 y_{0}(x), & \text { for } i=j\end{cases}
$$

where $\mathrm{N}_{\mathrm{i}}(\mathrm{x})$ is the normalization integral and the index $\mathrm{i}$ in equation (18b) can thus be dropped.

The eigenvalue problem defined by equation (15) allows for the definition of the following integral transform pair:

$$
\bar{\phi}_{\mathrm{i}}(\mathrm{x})=\frac{1}{\mathrm{~N}(\mathrm{x})} \int_{-\mathrm{y}_{1}}^{\mathrm{y}_{2}} \mathrm{Y}_{\mathrm{i}}(\mathrm{x}, \mathrm{y}) \phi(\mathrm{x}, \mathrm{y}) \mathrm{dy}, \quad \text { transform }
$$




$$
\phi(\mathrm{x}, \mathrm{y})=\sum_{\mathrm{i}=1}^{\infty} \mathrm{Y}_{\mathrm{i}}(\mathrm{x}, \mathrm{y}) \bar{\phi}_{\mathrm{i}}(\mathrm{x}), \quad \text { inverse }
$$

We can now accomplish the integral transformation of the original partial differential system given by equations (8)-(10). For this purpose, equation (8) is multiplied by $\mathrm{Y}_{\mathrm{i}}$ and is then integrated over the domain $\left[-\mathrm{y}_{1}(\mathrm{x}), \mathrm{y}_{2}(\mathrm{x})\right]$ in $\mathrm{y}$. After that, the inverse formula given by equation (20) is employed, resulting after some manipulations in the following coupled ordinary differential system for the calculation of the transformed potentials $\bar{\phi}_{i}$ :

\section{Integral transforms \\ solution}

$$
\begin{aligned}
\bar{\phi}_{\mathrm{i}}^{(\mathrm{iv})}= & -\mu_{\mathrm{i}}^{4} \bar{\phi}_{\mathrm{i}}+\frac{\mathrm{L}_{\mathrm{i}}}{\mathrm{N}}+\frac{\mathrm{Re}}{\mathrm{N}} \sum_{\mathrm{j}=1}^{\infty} \sum_{\mathrm{k}=1}^{\infty}\left\{\mathrm{A}_{\mathrm{ijk}} \bar{\phi}_{\mathrm{j}} \bar{\phi}_{\mathrm{k}}+\mathrm{B}_{\mathrm{ijk}} \bar{\phi}_{\mathrm{j}} \bar{\phi}_{\mathrm{k}}^{\prime}+\mathrm{C}_{\mathrm{ijk}} \bar{\phi}_{\mathrm{j}} \bar{\phi}_{\mathrm{k}}^{\prime \prime \prime}+\mathrm{D}_{\mathrm{ijk}} \bar{\phi}_{\mathrm{j}} \bar{\phi}_{\mathrm{k}}^{\prime \prime \prime}\right. \\
& \left.+\mathrm{E}_{\mathrm{ijk}} \bar{\phi}_{\mathrm{j}}^{\prime} \bar{\phi}_{\mathrm{k}}+\mathrm{F}_{\mathrm{ijk}} \bar{\phi}_{\mathrm{j}}^{\prime} \bar{\phi}_{\mathrm{k}}^{\prime}+\mathrm{G}_{\mathrm{ijk}} \bar{\phi}_{\mathrm{j}}^{\prime} \bar{\phi}_{\mathrm{k}}^{\prime \prime}\right\}+\frac{1}{\mathrm{~N}} \sum_{\mathrm{j}=1}^{\infty}\left\{\mathrm{H}_{\mathrm{ij}} \bar{\phi}_{\mathrm{j}}+\mathrm{I}_{\mathrm{ij}} \bar{\phi}_{\mathrm{j}}^{\prime}+\mathrm{J}_{\mathrm{ij}} \bar{\phi}_{\mathrm{j}}^{\prime \prime}+\mathrm{K}_{\mathrm{ij}} \bar{\phi}_{\mathrm{j}}^{\prime \prime \prime}\right\}
\end{aligned}
$$

The outflow boundary conditions are here chosen from two possibilities. In the first one, the duct is considered to be finite (truncated duct), and the following boundary conditions are employed:

$$
\mathrm{v}\left(\mathrm{x}_{\text {out }}, \mathrm{y}\right)=0 ; \quad \frac{\partial \omega\left(\mathrm{x}_{\text {out }}, \mathrm{y}\right)}{\partial \mathrm{x}}=0
$$

where $\omega$ is the vorticity.

The second possibility considers that the duct is infinite. Therefore, when $x \rightarrow \infty$, the outflow boundary conditions are those of a fully developed flow in a parallel-plates channel, which are given by:

$$
\mathrm{u}(\infty, \mathrm{y})=\frac{3}{2}\left(1-\mathrm{y}^{2}\right) ; \quad \mathrm{v}(\infty, \mathrm{y})=0
$$

The boundary conditions given by equations (22) and (23), after introducing the definition of the streamfunction (equation (6)) and the general filtering function given by equation (7), are rewritten as:

- For a truncated duct:

$$
\begin{gathered}
\frac{\partial \phi\left(x_{\text {out }}, y\right)}{\partial x}+\frac{\partial F\left(x_{\text {out }}, y\right)}{\partial x}=0 \\
\frac{\partial^{3} \phi\left(x_{\text {out }}, y\right)}{\partial x^{3}}+\frac{\partial^{3} \phi\left(x_{\text {out }}, y\right)}{\partial \mathrm{x}^{2} y^{2}}+\frac{\partial^{3} F\left(x_{\text {out }}, y\right)}{\partial x^{3}}+\frac{\partial^{3} F\left(x_{\text {out }}, y\right)}{\partial x \partial y^{2}}=0
\end{gathered}
$$

- For an infinite duct:

$$
\begin{gathered}
\phi(\infty, y)=0 \\
\frac{\partial \phi(\infty, y)}{\partial x}=0
\end{gathered}
$$


$\mathrm{HFF}$

21,2

226
Now, the integral transformation process of equations (24) and (25) is similar to that of obtaining equation (21), i.e. the equations are multiplied by $Y_{i}$ and then integrated over the domain $\left[-\mathrm{y}_{1}(\mathrm{x}), \mathrm{y}_{2}(\mathrm{x})\right]$ in $\mathrm{y}$. After that, the inverse formula given by equation (20) is employed, yielding:

- For a truncated duct:

$$
\begin{gathered}
\bar{\phi}_{\mathrm{i}}(0)=0 ; \quad \frac{\mathrm{d} \bar{\phi}_{\mathrm{i}}(0)}{\mathrm{dx}}=0 \\
\frac{\mathrm{d} \bar{\phi}_{\mathrm{i}}\left(\mathrm{x}_{\text {out }}\right)}{\mathrm{dx}}=-\frac{1}{\mathrm{~N}\left(\mathrm{x}_{\text {out }}\right)}\left[\mathrm{M}_{\mathrm{i}}+\sum_{\mathrm{j}=1}^{\infty} \mathrm{N}_{\mathrm{ij}} \bar{\phi}_{\mathrm{j}}\left(\mathrm{x}_{\text {out }}\right)\right] \\
\frac{\mathrm{d}^{3} \bar{\phi}_{\mathrm{i}}\left(\mathrm{x}_{\text {out }}\right)}{\mathrm{dx}^{3}}=-\frac{1}{\mathrm{~N}\left(\mathrm{x}_{\text {out }}\right)}\left\{\mathrm{O}_{\mathrm{i}}+\sum_{\mathrm{j}=1}^{\infty}\left[\mathrm{P}_{\mathrm{ij}} \bar{\phi}_{\mathrm{j}}\left(\mathrm{x}_{\text {out }}\right)+\mathrm{Q}_{\mathrm{ij}} \frac{\mathrm{d} \bar{\phi}_{\mathrm{j}}\left(\mathrm{x}_{\text {out }}\right)}{\mathrm{dx}}+\mathrm{R}_{\mathrm{ij}} \frac{\mathrm{d}^{2} \bar{\phi}_{\mathrm{j}}\left(\mathrm{x}_{\text {out }}\right)}{\mathrm{dx} \mathrm{x}^{2}}\right]\right\}
\end{gathered}
$$

- For an infinite duct:

$$
\bar{\phi}_{\mathrm{i}}(0)=0 ; \quad \frac{\mathrm{d} \bar{\phi}_{\mathrm{i}}(0)}{\mathrm{dx}}=0 ; \quad \bar{\phi}_{\mathrm{i}}(\infty)=0 ; \quad \frac{\mathrm{d} \bar{\phi}_{\mathrm{i}}(\infty)}{\mathrm{dx}}=0
$$

The coefficients that depend on $\mathrm{x}$ are calculated from:

$$
\begin{gathered}
A_{i j k}=\int_{-y_{1}}^{y_{2}} Y_{i}\left[\frac{\partial Y_{j}}{\partial y}\left(\frac{\partial^{3} Y_{k}}{\partial x^{3}}+\frac{\partial^{3} Y_{k}}{\partial x \partial y^{2}}\right)-\frac{\partial Y_{j}}{\partial x}\left(\frac{\partial^{3} Y_{k}}{\partial x^{2} \partial y}+\frac{\partial^{3} Y_{k}}{\partial y^{3}}\right)\right] d y \\
B_{i j k}=\int_{-y_{1}}^{y_{2}} Y_{i}\left[\frac{\partial Y_{j}}{\partial y}\left(3 \frac{\partial^{2} Y_{k}}{\partial x^{2}}+\frac{\partial^{2} Y_{k}}{\partial y^{2}}\right)-2 \frac{\partial Y_{j}}{\partial x} \frac{\partial^{2} Y_{k}}{\partial x \partial y}\right] d y \\
C_{i j k}=\int_{-y_{1}}^{y_{2}} Y_{i}\left[3 \frac{\partial Y_{j}}{\partial y} \frac{\partial Y_{k}}{\partial x}-\frac{\partial Y_{j}}{\partial x} \frac{\partial Y_{k}}{\partial y}\right] d y \\
D_{i j k}=\int_{-y_{1}}^{y_{2}} Y_{i} \frac{\partial Y_{j}}{\partial y} Y_{k} d y ; \quad E_{i j k}=-\int_{-y_{1}}^{y_{2}} Y_{i} Y_{j}\left[\frac{\partial^{3} Y_{k}}{\partial x^{2} \partial y}+\frac{\partial^{3} Y_{k}}{\partial y^{3}}\right] d y \\
F_{i j k}=-2 \int_{-y_{1}}^{y_{2}} Y_{i} Y_{j} \frac{\partial^{2} Y_{k}}{\partial x \partial y} d y \quad G_{i j k}=-\int_{-y_{1}}^{y_{2}} Y_{i} Y_{j} \frac{\partial Y_{k}}{\partial y} d y \\
H_{i j}=a_{i j} R e-b_{i j} ; \quad I_{i j}=c_{i j} R e-d_{i j} ; \quad J_{i j}=e_{i j} R e-f_{i j} ; \\
K_{i j}=g_{i j} R e-h_{i j} ; \quad L_{i}=i_{i} R e-j_{i} \\
M_{i}=\int_{-y_{1}}^{y_{2}} Y_{i} \frac{\partial F}{\partial x} d y ; \quad N_{i j}=\int_{-y_{1}}^{y_{2}} Y_{i} \frac{\partial Y_{j}}{\partial x} d y
\end{gathered}
$$




$$
\begin{aligned}
& \mathrm{O}_{\mathrm{i}}=\int_{-\mathrm{y}_{1}}^{\mathrm{y}_{2}} \mathrm{Y}_{\mathrm{i}}\left[\frac{\partial^{3} \mathrm{~F}}{\partial \mathrm{x}^{3}}+\frac{\partial^{3} \mathrm{~F}}{\partial \mathrm{x} \partial \mathrm{y}^{2}}\right] d y ; \quad \mathrm{P}_{\mathrm{ij}}=\int_{-\mathrm{y}_{1}}^{\mathrm{y}_{2}} \mathrm{Y}_{\mathrm{i}}\left[\frac{\partial^{3} \mathrm{Y}_{\mathrm{j}}}{\partial \mathrm{x}^{3}}+\frac{\partial^{3} \mathrm{Y}_{\mathrm{j}}}{\partial \mathrm{x} \partial \mathrm{y}^{2}}\right] \mathrm{dy} \\
& Q_{i j}=\int_{-y_{1}}^{y_{2}} Y_{i}\left[3 \frac{\partial^{2} Y_{j}}{\partial x^{2}}+\frac{\partial^{2} Y_{j}}{\partial y^{2}}\right] d y ; \quad R_{i j}=3 \int_{-y_{1}}^{y_{2}} Y_{i} \frac{\partial Y_{j}}{\partial x} d y \\
& a_{i j}=\int_{-y_{1}}^{y_{2}} Y_{i}\left[\frac{\partial Y_{j}}{\partial y}\left(\frac{\partial^{3} F}{\partial x^{3}}+\frac{\partial^{3} F}{\partial x \partial y^{2}}\right)-\frac{\partial Y_{j}}{\partial x}\left(\frac{\partial^{3} F}{\partial x^{2} \partial y}+\frac{\partial^{3} F}{\partial y^{3}}\right)\right. \\
& \left.+\left(\frac{\partial^{3} Y_{j}}{\partial x^{3}}+\frac{\partial^{3} Y_{j}}{\partial x \partial y^{2}}\right) \frac{\partial F}{\partial y}-\left(\frac{\partial^{3} Y_{j}}{\partial x^{2} \partial y}+\frac{\partial^{3} Y_{j}}{\partial y^{3}}\right) \frac{\partial F}{\partial x}\right] d y \\
& b_{i j}=\int_{-y_{1}}^{y_{2}} Y_{i}\left[\frac{\partial^{4} Y_{j}}{\partial x^{4}}+2 \frac{\partial^{4} Y_{j}}{\partial x^{2} \partial y^{2}}\right] d y \\
& c_{i j}=\int_{-y_{1}}^{y_{2}} Y_{i}\left[Y_{j}\left(-\frac{\partial^{3} F}{\partial x^{2} \partial y}-\frac{\partial^{3} F}{\partial y^{3}}\right)+\left(3 \frac{\partial^{2} Y_{j}}{\partial x^{2}}+\frac{\partial^{2} Y_{j}}{\partial y^{2}}\right) \frac{\partial F}{\partial y}-2 \frac{\partial^{2} Y_{j}}{\partial x \partial y} \frac{\partial F}{\partial x}\right] d y \\
& d_{i j}=4 \int_{-y_{1}}^{y_{2}} Y_{i}\left[\frac{\partial^{3} Y_{j}}{\partial x^{3}}+\frac{\partial^{3} Y_{j}}{\partial x \partial y^{2}}\right] d y ; \quad e_{i j}=\int_{-y_{1}}^{y_{2}} Y_{i}\left[3 \frac{\partial Y_{j}}{\partial x} \frac{\partial F}{\partial y}-\frac{\partial Y_{j}}{\partial y} \frac{\partial F}{\partial x}\right] d y \\
& \mathrm{f}_{\mathrm{ij}}=2 \int_{-\mathrm{y}_{1}}^{\mathrm{y}_{2}} \mathrm{Y}_{\mathrm{i}}\left[3 \frac{\partial^{2} \mathrm{Y}_{\mathrm{j}}}{\partial \mathrm{x}^{2}}+\frac{\partial^{2} \mathrm{Y}_{\mathrm{j}}}{\partial \mathrm{y}^{2}}\right] \mathrm{dy} ; \quad \mathrm{g}_{\mathrm{ij}}=\int_{-\mathrm{y}_{1}}^{\mathrm{y}_{2}} \mathrm{Y}_{\mathrm{i}} \mathrm{Y}_{\mathrm{j}} \frac{\partial \mathrm{F}}{\partial \mathrm{y}} \mathrm{dy} \\
& h_{i j}=4 \int_{-y_{1}}^{y_{2}} Y_{i} \frac{\partial Y_{j}}{\partial x} d y \\
& i_{i}=\int_{-y_{1}}^{y_{2}} Y_{i}\left(\frac{\partial F}{\partial y} \frac{\partial^{3} F}{\partial x^{3}}+\frac{\partial F}{\partial y} \frac{\partial^{3} F}{\partial x \partial y^{2}}-\frac{\partial F}{\partial x} \frac{\partial^{3} F}{\partial x^{2} \partial y}-\frac{\partial F}{\partial x} \frac{\partial^{3} F}{\partial y^{3}}\right) d y \\
& \mathrm{j}_{\mathrm{i}}=\int_{-\mathrm{y}_{1}}^{\mathrm{y}_{2}} \mathrm{Y}_{\mathrm{i}}\left(\frac{\partial^{4} \mathrm{~F}}{\partial \mathrm{x}^{4}}+2 \frac{\partial^{4} \mathrm{~F}}{\partial \mathrm{x}^{2} \partial \mathrm{y}^{2}}\right) d y
\end{aligned}
$$

For computational purposes, it is necessary to truncate the infinite expansions in a sufficiently large number of terms so as to achieve the user prescribed relative error target for obtaining the original potentials, in this case the streamfunction values, where NTV is here the order of truncation of the infinite series. Also, in order to solve the transformed ODE system, efficient numerical algorithms for boundary value problems are to be employed, such as the subroutine DBVPFD from the IMSL Library (1991), which offers an automatic adaptive scheme for local error control of the numerical results for the transformed potentials. It is then necessary to rewrite the transformed ODE system as a first order one, by introducing the following dependent variables: 
$\mathrm{HFF}$

21,2

$$
\begin{gathered}
\chi_{\mathrm{i}}=\bar{\phi}_{\mathrm{i}} ; \quad \frac{\mathrm{d} \chi_{\mathrm{i}}}{\mathrm{dx}}=\chi_{\mathrm{NTV}+\mathrm{i}}=\frac{\mathrm{d} \bar{\phi}_{\mathrm{i}}}{\mathrm{dx}} ; \quad \frac{\mathrm{d} \chi_{\mathrm{NTV}+\mathrm{i}}}{\mathrm{dx}}=\chi_{2} \mathrm{NTV+i}=\frac{\mathrm{d}^{2} \bar{\phi}_{\mathrm{i}}}{\mathrm{dx}^{2}} \\
\frac{\mathrm{d} \chi_{2 \mathrm{NTV}+\mathrm{i}}}{\mathrm{dx}}=\chi_{3 \mathrm{NTV}+\mathrm{i}}=\frac{\mathrm{d}^{3} \bar{\phi}_{\mathrm{i}}}{\mathrm{dx}^{3}} ; \quad \frac{\mathrm{d} \chi_{3 \mathrm{NTV}+\mathrm{i}}}{\mathrm{dx}}=\frac{\mathrm{d}^{4} \bar{\phi}_{\mathrm{i}}}{\mathrm{dx}^{4}}
\end{gathered}
$$

Therefore, by making use of equations (30), the transformed system can be rewritten as:

$$
\begin{gathered}
\frac{\mathrm{d} \chi_{\mathrm{i}}}{\mathrm{d} \tau}=\frac{\chi_{\mathrm{NTV}+\mathrm{i}}}{(\mathrm{d} \tau / \mathrm{dx})} ; \quad \frac{\mathrm{d} \chi_{\mathrm{NTV}+\mathrm{i}}}{\mathrm{d} \tau}=\frac{\chi_{2} \mathrm{NTV}+\mathrm{i}}{(\mathrm{d} \tau / \mathrm{dx})} ; \quad \frac{\mathrm{d} \chi_{2} \mathrm{NTV}+\mathrm{i}}{\mathrm{d} \tau}=\frac{\chi_{3} \mathrm{NTV}+\mathrm{i}}{(\mathrm{d} \tau / \mathrm{dx})} \\
\frac{\mathrm{d} \chi_{3 \mathrm{NTV}+\mathrm{i}}}{\mathrm{d} \tau}=\frac{\left.\begin{array}{l}
-\mu_{\mathrm{i}}^{4} \chi_{\mathrm{i}}+\frac{\mathrm{L}_{\mathrm{i}}}{\mathrm{N}}+\frac{\mathrm{Re}}{\mathrm{N}} \sum_{\mathrm{j}=1}^{\mathrm{NTV}} \sum_{\mathrm{k}=1}^{\mathrm{NTV}}\left[\mathrm{A}_{\mathrm{ijk}} \chi_{\mathrm{j}} \chi_{\mathrm{k}}+\mathrm{B}_{\mathrm{ijk}} \chi_{\mathrm{j}} \chi_{\mathrm{NTV}+\mathrm{k}}\right. \\
+\mathrm{C}_{\mathrm{ijk}} \chi_{\mathrm{j}} \chi_{2 \mathrm{NTV}+\mathrm{k}}+\mathrm{D}_{\mathrm{ijk}} \chi_{\mathrm{j}} \chi_{3} \mathrm{NTV+ \textrm {k }}+\mathrm{E}_{\mathrm{ijk}} \chi_{\mathrm{NTV}+\mathrm{j} \chi_{\mathrm{k}}} \\
\left.+\mathrm{F}_{\mathrm{ijk}} \chi_{\mathrm{NTV}+\mathrm{j}} \chi_{\mathrm{NTV}+\mathrm{k}}+\mathrm{G}_{\mathrm{ijk}} \chi_{\mathrm{NTV}+\mathrm{j}} \chi_{2 \mathrm{NTV}+\mathrm{k}}\right] \\
+\frac{1}{\mathrm{~N}} \sum_{\mathrm{j}=1}^{\mathrm{NTV}}\left[\mathrm{H}_{\mathrm{ij}} \chi_{\mathrm{j}}+\mathrm{I}_{\mathrm{ij}} \chi_{\mathrm{NTV}+\mathrm{j}}+\mathrm{J}_{\mathrm{ij}} \chi_{2 \mathrm{NTV}+\mathrm{j}}+\mathrm{K}_{\mathrm{ij}} \chi_{3 \mathrm{NTV}+\mathrm{j}}\right]
\end{array}\right\}}{(\mathrm{d} \tau / \mathrm{dx})},
\end{gathered}
$$

for $\mathrm{i}=1,2, \ldots, \mathrm{NTV}$

with $\tau=1-\mathrm{e}-{ }^{\mathrm{cx}}, 0 \leq \tau \leq 1$, and c being a parameter of scale compression for the case of an infinite duct.

Analyzing the ODE system given by equations (31), we observe that the integral coefficients depend on the axial position $\mathrm{x}$. This would imply in a high-computational cost if the coefficients would require error-controlled numerical integrations, once they need to be continuously reevaluated along the solution procedure for the ordinary differential equations system. However, all of them could be analytically determined through symbolic computation (Wolfram, 1999). Also, the computational procedure is organized in such a way that the x-independent portions of each coefficient are calculated only once, before entering the boundary-value problem solver, and stored. Along the ODE system integration, they are then recalled and multiplied by the functions that take into account the dependence of the irregular domain as the differential system is being numerically solved.

\section{Results and discussion}

We analyze the wavy wall duct whose geometry was considered in Wang and Chen (2002) and is shown schematically in Figure 2. The functions that describe this symmetric geometry in dimensionless terms are given as:

$$
\mathrm{y}_{1}(\mathrm{x})=1+\mathrm{f}(\mathrm{x}) ; \quad \mathrm{y}_{2}(\mathrm{x})=1+\mathrm{f}(\mathrm{x}) ; \quad \mathrm{f}(\mathrm{x})=\alpha \sin [\pi(\mathrm{x}-3)]
$$

where $\alpha=\mathrm{a}^{*} / \mathrm{b}$ is the dimensionless amplitude of the wavy surface, and the value of the axial coordinate at the duct outlet $\mathrm{x}_{\text {out }}$ was taken as $\mathrm{x}_{\text {out }}=20$. In the present analysis, the interval used for the axial coordinate $\mathrm{x}$ was $3 \leq \mathrm{x} \leq 15$, which corresponds to six complete sinusoidal waves. 
Tables I-III show the convergence of the streamfunction values along the line $\mathrm{y}=0.5$ for $\operatorname{Re}=100$ and $\alpha=0.3, \operatorname{Re}=300$ and $\alpha=0.2$ and $\operatorname{Re}=500$ and $\alpha=0.1$, respectively, for the two cases of outflow boundary conditions (here named truncated duct and infinite duct, respectively). For the case of $\operatorname{Re}=100$ and $\alpha=0.3$, it is observed that full convergence to four significant digits is achieved with $\mathrm{NTV}=18$, while for the case $\operatorname{Re}=500$ and $\alpha=0.1$, similar convergence is reached only with $\mathrm{NTV}=22$. In Table II, the results obtained with NTV $=22$ and 26 have remained unaffected, and the same happens in Table III, though this is not evident from the previous column for $\mathrm{NTV}=18$. The algorithm is organized so that numerical computation automatically ceases once the requested precision is achieved. In general, the results for the two cases of outflow boundary conditions analyzed are in perfect agreement, with a slight difference for axial positions near the duct outlet $(\mathrm{x}=20)$, which in terms of relative deviation is always below 0.5 percent for the fully converged results $(\mathrm{NTV}=22)$. This is justified by the fact that for the infinite duct, the fully developed region is not imposed and is reached at axial positions a little further away.

Figures 3(a)-(d) show a comparison of the present results for the product fRe with those numerically obtained by Wang and Chen (2002) at different axial positions along the channel, for the cases $\operatorname{Re}=100$ and $\alpha=0.2, \operatorname{Re}=300$ and $\alpha=0.2, \operatorname{Re}=500$ and $\alpha=0.1$ and $\operatorname{Re}=500$ and $\alpha=0.2$, respectively. Wang and Chen (2002) employed a coordinate transformation and the spline alternating-direction implicit method, an improved version of the cubic spline collocation method. One can see an excellent agreement among the four sets of results, obtained via two quite different solution methodologies, offering a fairly reliable co-validation report. Also, it can be verified how importantly the Reynolds number influences the product fRe, i.e. higher peaks are found for increasing Reynolds numbers and fixed duct geometry, indicating the evidently expected higher viscous effects in the flow. In both cases, the GITT results for the product fRe are already converged to the graph scale. In tabular form, a convergence rate of at least two significant digits for fRe is observed in most cases. The computation of the friction factor involves derivatives of the velocity field, which present a slower convergence behavior than the original expanded potentials. Also, one can notice negative values for the product fRe in some positions, which indicate the presence of recirculation zones. In addition, Figure 4 shows the convergence behavior of the product fRe for the most severe case of $\operatorname{Re}=500$ and $\alpha=0.3$, with different truncations orders $\mathrm{NTV}=13,15,17$ and 19, in order to demonstrate the graphical convergence behavior of such results. This case brings a combination of the highest Reynolds number and duct amplitude considered in the present work; nevertheless, an excellent graphical

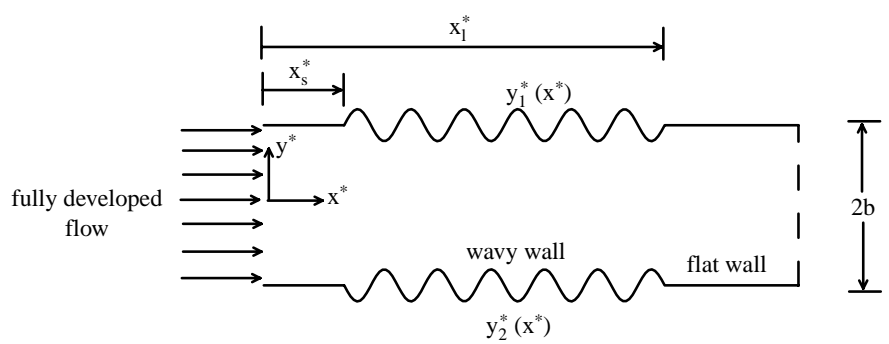

Figure 2. Geometric and flow characteristics of the wavy wall duct analyzed 


\section{$\mathrm{HFF}$ \\ 21,2}

\section{0}

\section{Table I.}

Convergence behavior of the streamfunction at $\mathrm{y}=0.5$ for $\operatorname{Re}=100$ and $\alpha=0.3$

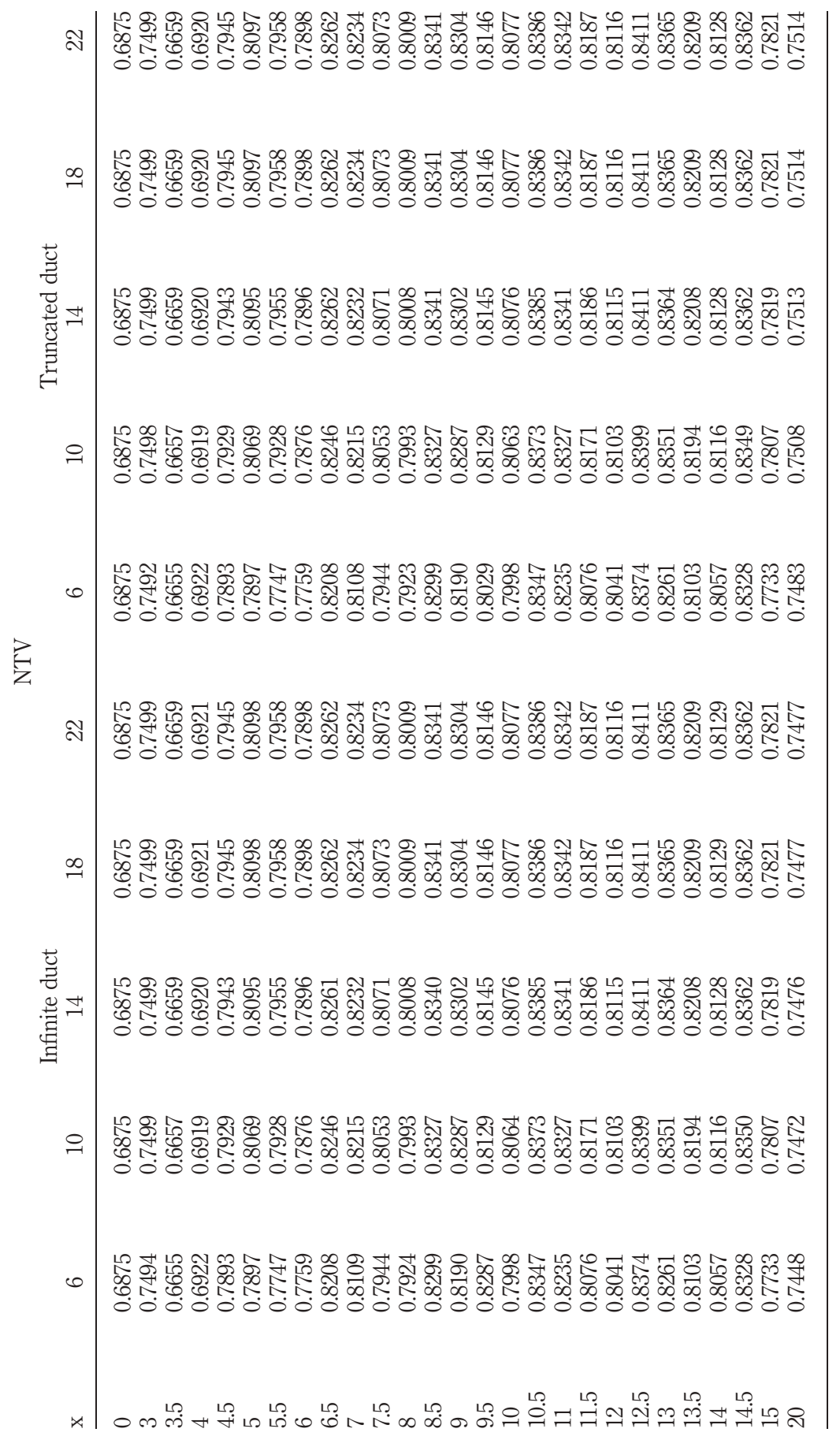




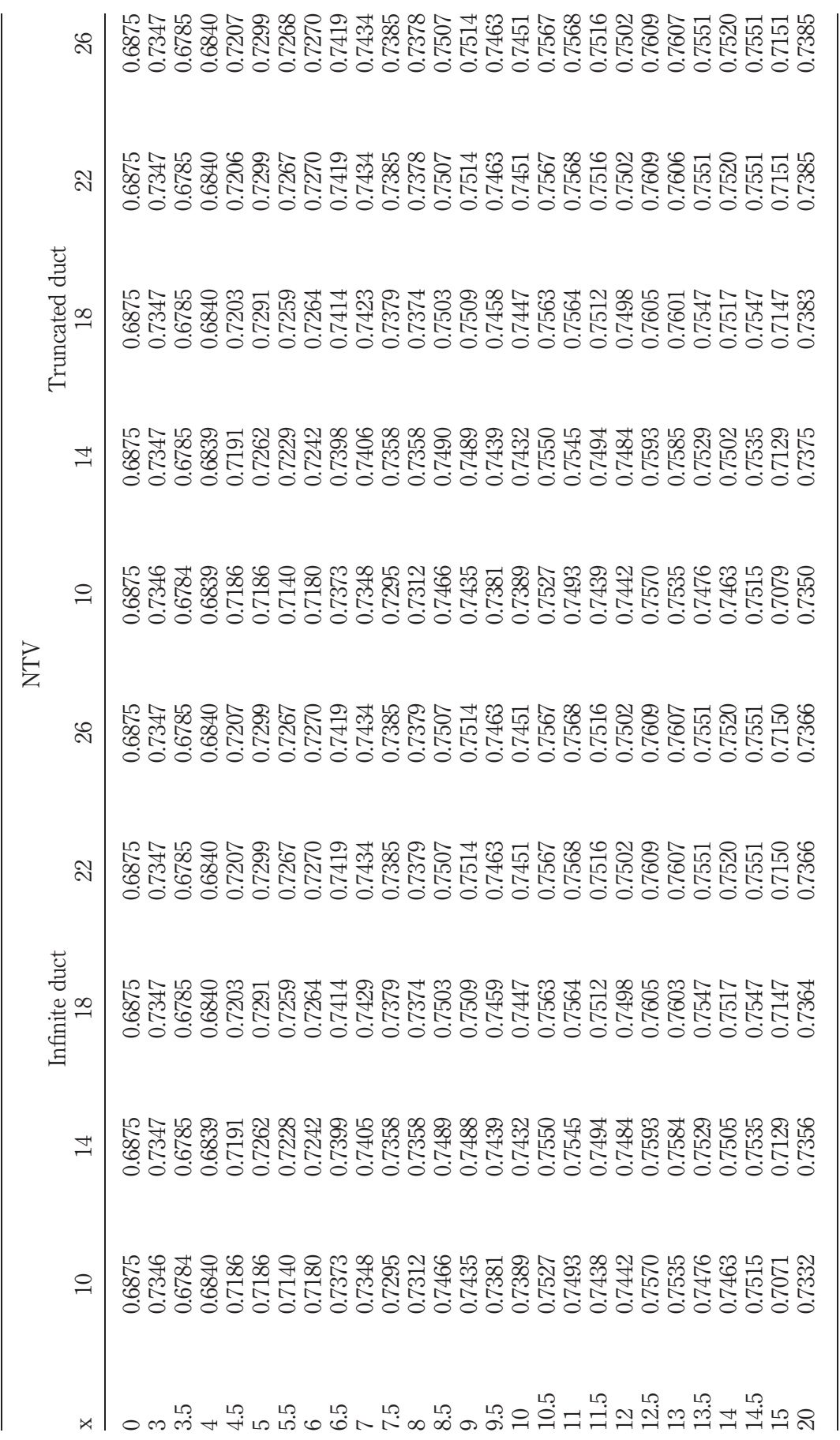

231

Table II.

Convergence behavior of the streamfunction at $\mathrm{y}=0.5$ for $\mathrm{Re}=300$ and 


\section{$\mathrm{HFF}$ \\ 21,2}

232

Table III.

Convergence behavior of the streamfunction at $\mathrm{y}=0.5$ for $\mathrm{Re}=500$ and $\alpha=0.1$

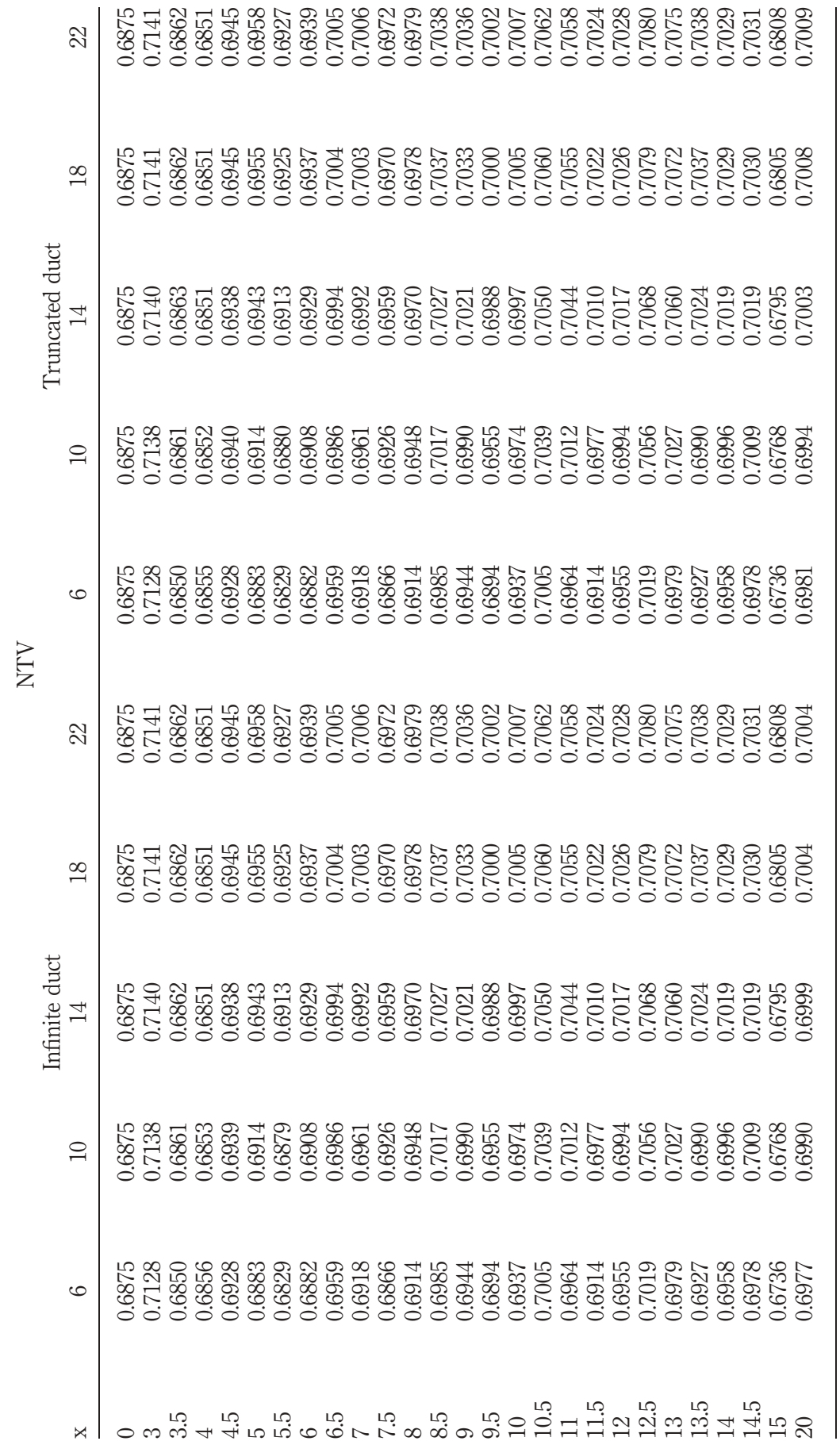




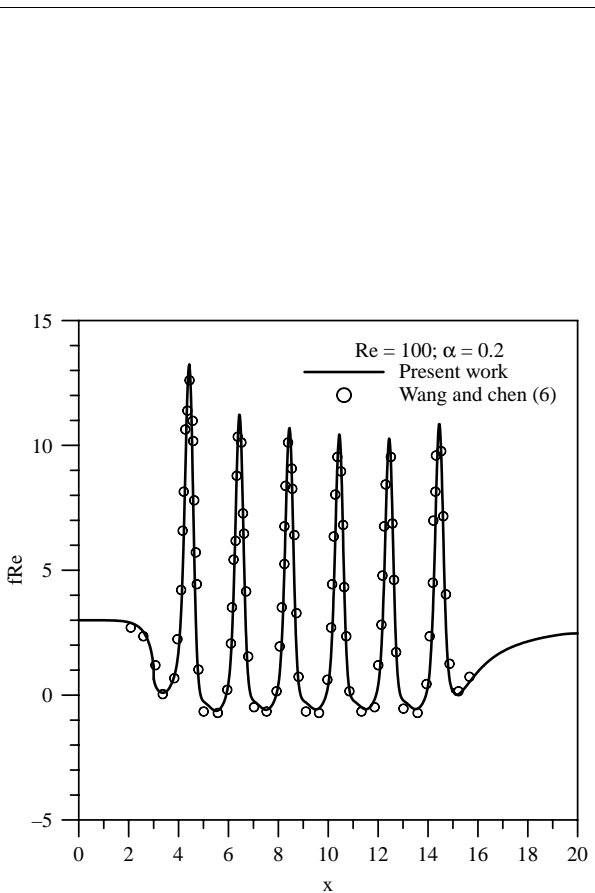

(a)

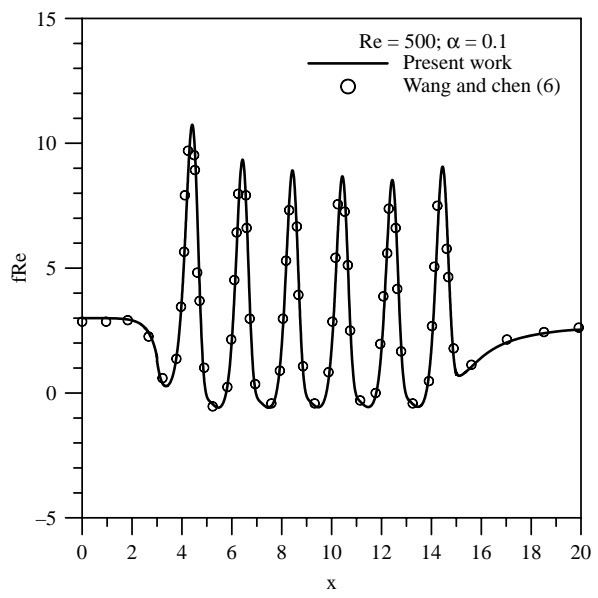

(c)

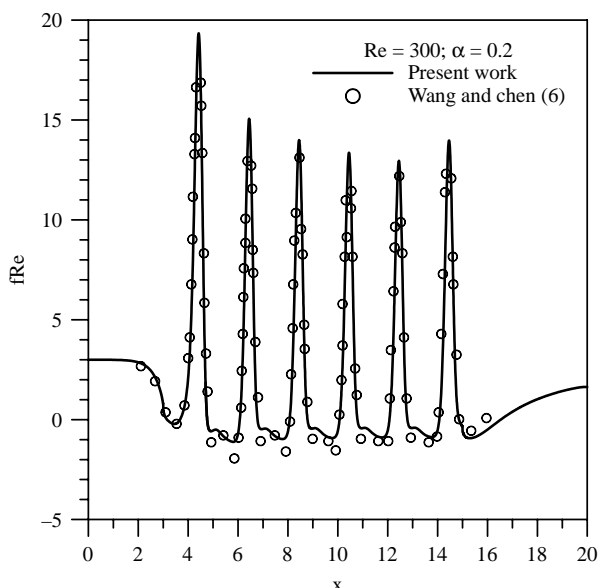

(b)

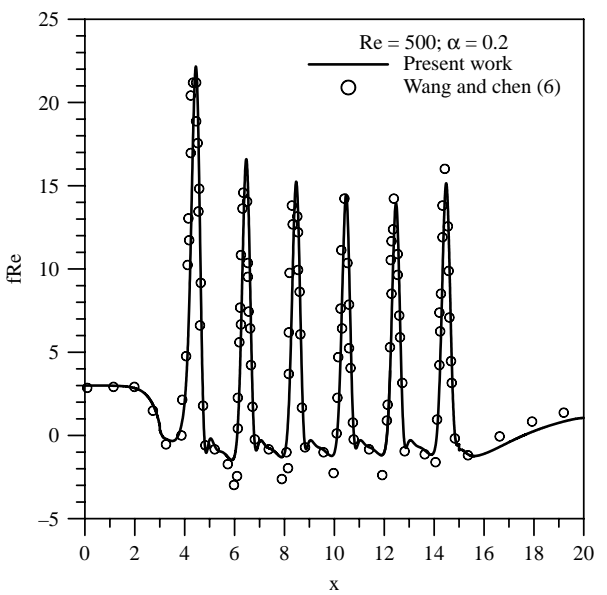

(d)

Notes: (a) $\operatorname{Re}=100$ and $\alpha=0.2$; (b) $\operatorname{Re}=300$ and $\alpha=0.2$; (c) $\operatorname{Re}=500$ and $\alpha=0.1$; (d) $\mathrm{R}=500$ and $\alpha=0.2$

Integral transforms

solution

233

Figure 3.

Comparison of GITT results for the product fRe for different values of the

Reynolds number and dimensionless duct amplitude, against numerical results of spline alternating-direction implicit method of Wang and Chen (2002) 


\section{HFF}

21,2

\section{4}

Figure 4.

Convergence behavior of the distribution of the product fRe for $\operatorname{Re}=500$ and $\alpha=0.3$

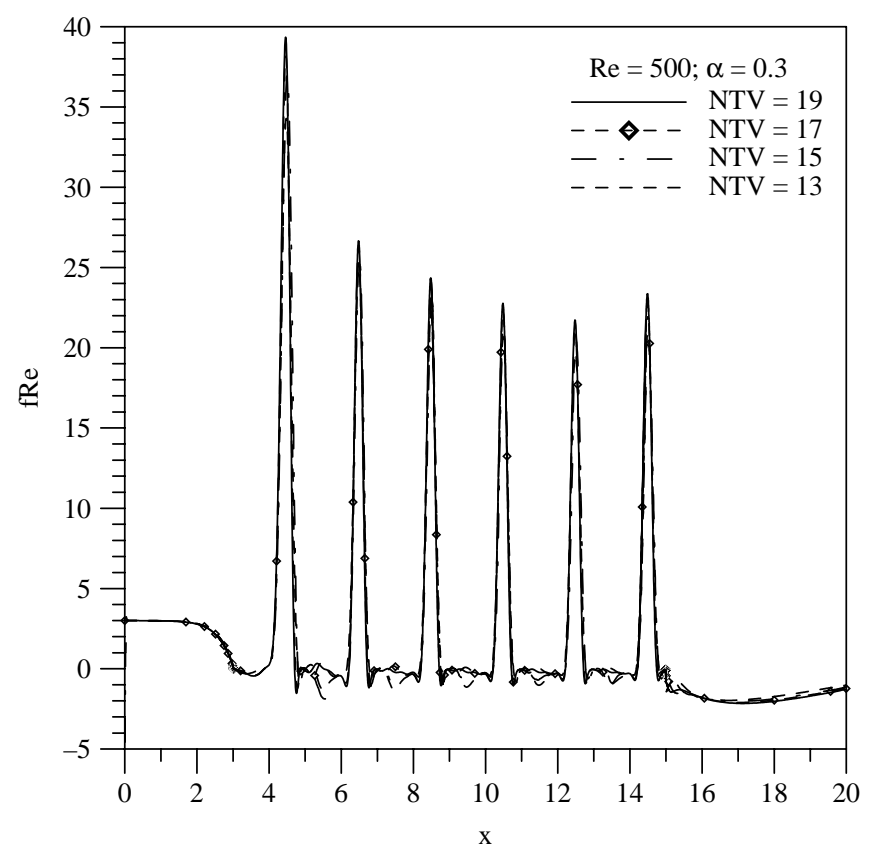

convergence of fRe is also verified for this situation. Figure 5(a)-(c) shows the product fRe for different values of the Reynolds number, respectively, $\operatorname{Re}=100,300$ and 500, and varying the dimensionless duct amplitude for each set of curves, so as to illustrate the effect of the duct geometry. Clearly, the fRe peaks are markedly affected by the increase in the amplitude of the sinusoidal oscillation on the boundary geometry, with the growing recirculation flow patterns, as will be more evident from the contour plots for the streamfunction in what follows.

As for the product fRe, Figure 6 shows the convergence behavior for the evolution of the axial component velocity at the duct centerline, $\mathrm{u}(\mathrm{x}, 0)$, for the more severe case of $\operatorname{Re}=500$ and $\alpha=0.3$, and again a reasonable graphical convergence rate is achieved for this parameter. Then, Figure 7(a)-(c) shows the evolution of the axial component velocity at the duct centerline for different values of the dimensionless duct amplitude in each set of curves for $\mathrm{Re}=100,300$ and 500, respectively. As the Reynolds number and the duct amplitude increase, it is observed higher distortions in the longitudinal velocity component evolutions, demonstrating the increased perturbation on the core flow around the channel centerline. With the increase on the channel walls sinusoidal amplitude, the flow is noticeably accelerated in average terms along the centerline within this development region, as a direct result of the effective flow path periodic constriction.

Finally, Figures 8(a)-(c)-10(a)-(c) show the isolines patterns of the streamfunction for all three values of Reynolds numbers and duct wall sinusoidal amplitudes here considered. The marked influence of the combined increase on Reynolds number and channel amplitude is clearly observable in the appearance of stronger recirculation 


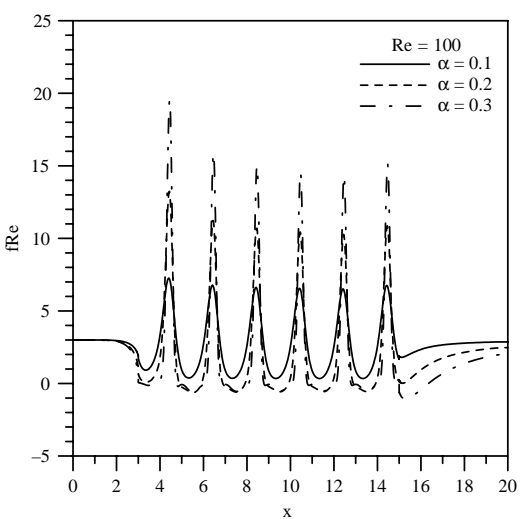

(a)

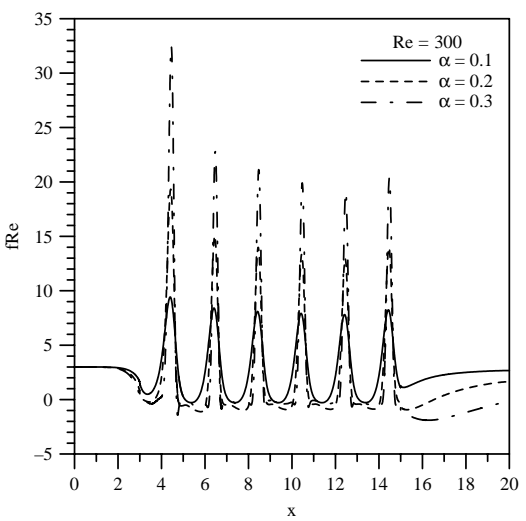

(b)

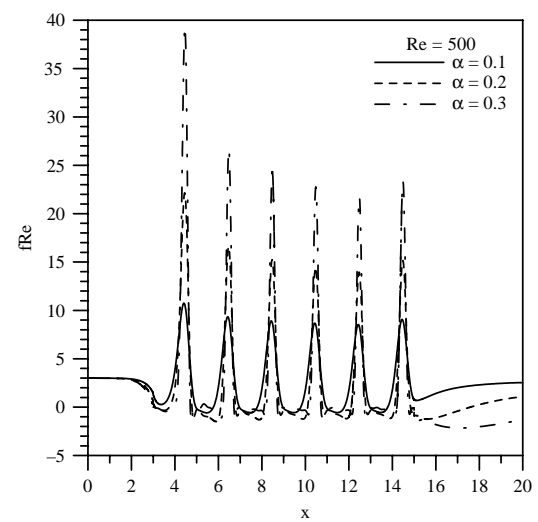

(c)

Notes: (a) $\operatorname{Re}=100$; (b) $\operatorname{Re}=300$; (c) $\operatorname{Re}=500$

\section{Integral transforms solution}

235

Figure 5.

Profiles of the product fRe for different values of the Reynolds number and dimensionless duct amplitude 


\section{HFF}

\section{1,2}

\section{6}

Figure 6.

Convergence behavior of the evolution of the axial component velocity at the duct centerline for $\operatorname{Re}=500$ and $\alpha=0.3$

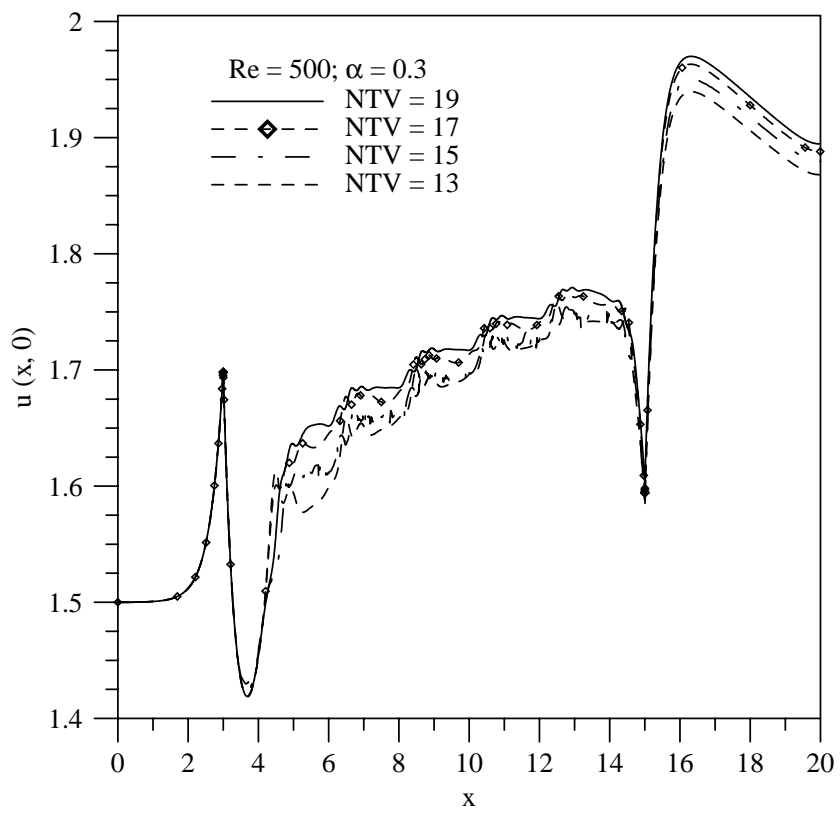

zones internally to the "cavities" formed by the wavy walls. Specifically, the case of $\operatorname{Re}=500$ and $\alpha=0.3$ shows the strongest recirculation zone, even at the duct outlet, demonstrating the influence pointed out above.

\section{Conclusions}

The GITT has been demonstrated in the hybrid numerical-analytical solution of laminar flow problems within channels with wavy walls. The case of a wavy walls duct has been more closely considered in light of its importance in heat-transfer enhancement applications. A steady two-dimensional formulation based on the Navier-Stokes equations and on the streamfunction definition is adopted. Employing a simple coordinate transformation, a straightforward filtering solution is then obtained, offering a relevant convergence enhancement effect in the eigenfunction expansion for the streamfunction.

The convergence behavior of the proposed eigenfunction expansion is illustrated, and numerical results for the friction factor are critically compared with previously obtained numerical results from discrete approaches, with good agreement. A few additional results are also presented and employed in the interpretation of some physical aspects in this flow problem.

The proposed hybrid approach is fairly general and opens up several possibilities of analysis, including the search of optimized heat-transfer surfaces forms under prescribed pressure drop requirements. Since the hybrid solution is fully analytical in the transversal direction, integrals and derivatives at any cross-section can be readily derived without further numerical involvement. The approach can also be quite interesting in the analysis of periodic fully developed situations. 


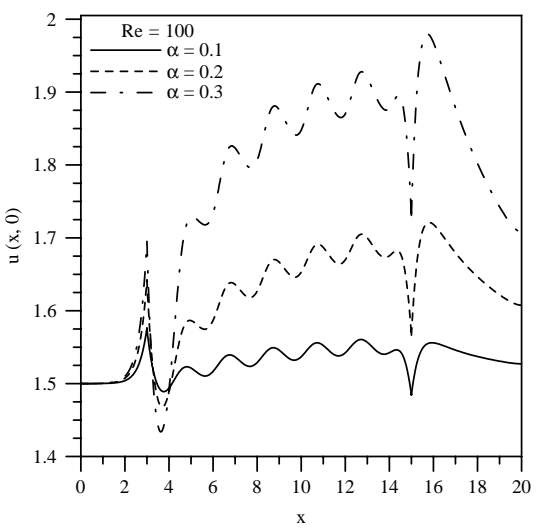

\section{Integral transforms solution}

(a)

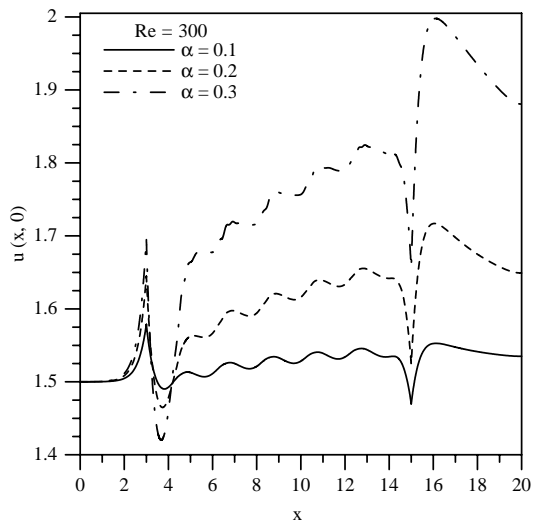

(b)

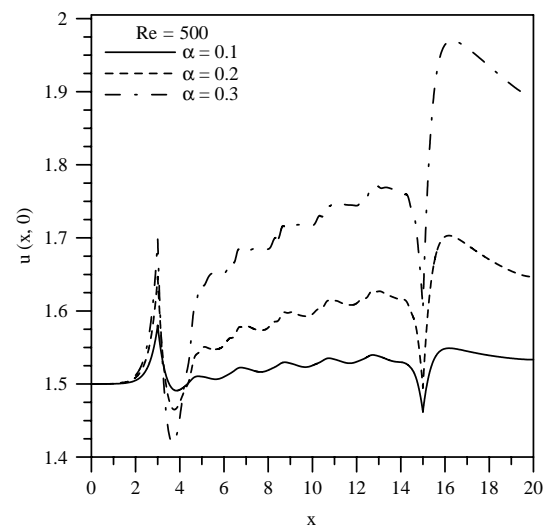

(c)

Notes: (a) $\operatorname{Re}=100$; (b) $\operatorname{Re}=300$; (c) $\operatorname{Re}=500$

Figure 7.

Evolution of the axial component velocity at the duct centerline for different values of the Reynolds number and dimensionless duct amplitude 


\section{$\mathrm{HFF}$}

21,2

\section{8}

Figure 8.

Streamline patterns along the duct length for different dimensionless duct amplitude and $\operatorname{Re}=100$

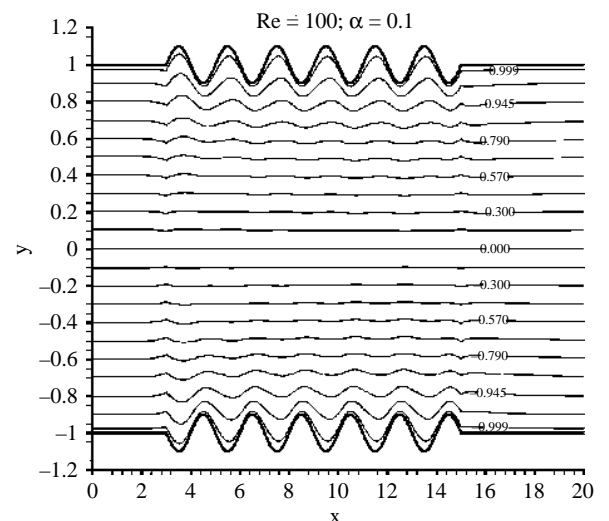

(a)

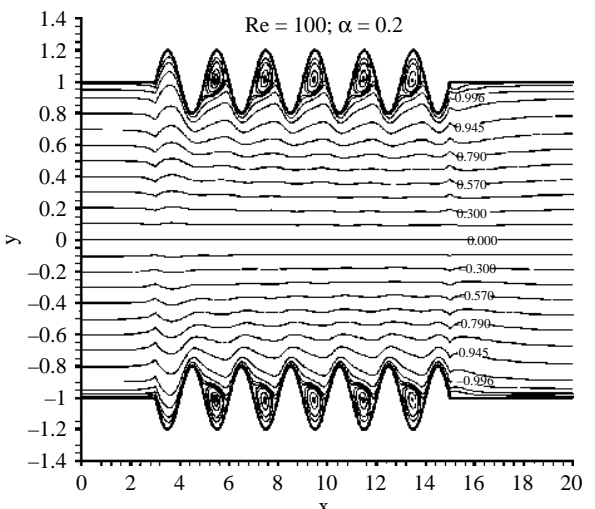

(b)

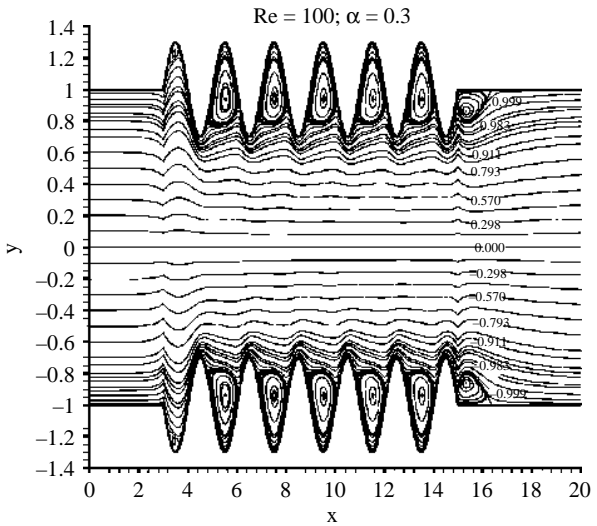

(c)

Notes: (a) $\mathrm{a}=0.1$; (b) $\mathrm{a}=0.2 ;$ (c) $\mathrm{a}=0.3$ 


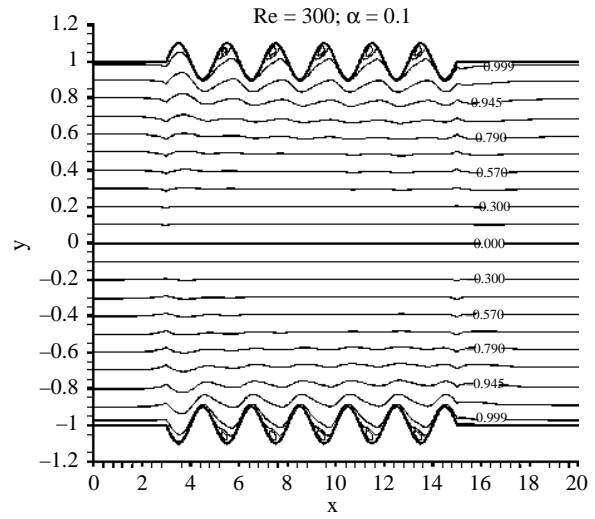

(a)

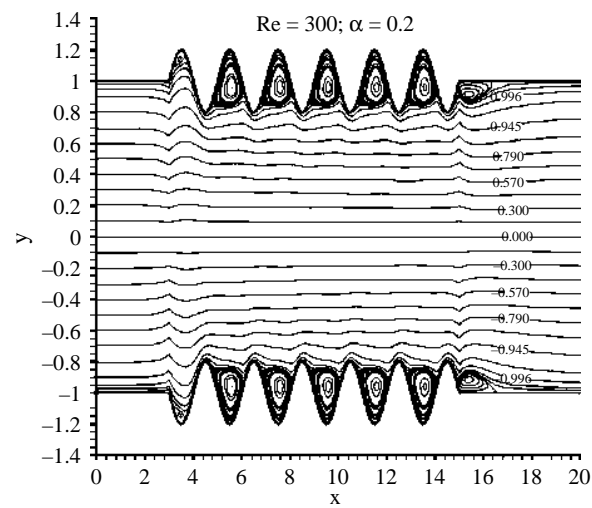

(b)

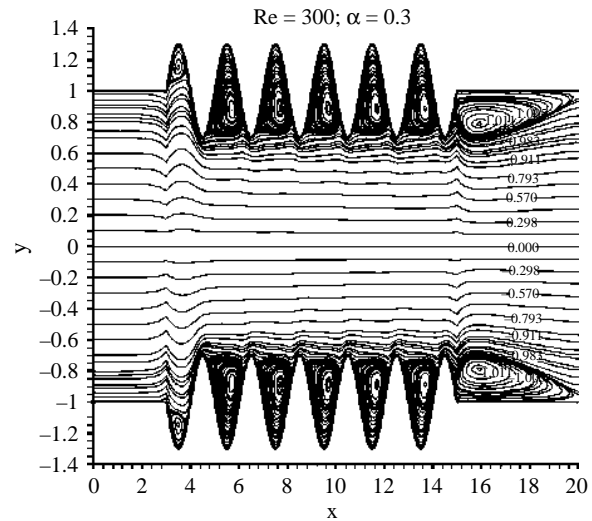

(c)

Notes: (a) $\alpha=0.1$; (b) $\alpha=0.2$; (c) $\alpha=0.3$
Integral transforms solution

239
Figure 9.

Streamline patterns along the duct length for different dimensionless duct amplitude and $\operatorname{Re}=300$ 
$\mathrm{HFF}$

21,2

240

Figure 10.

Streamline patterns along the duct length for different dimensionless duct amplitude and $\operatorname{Re}=500$

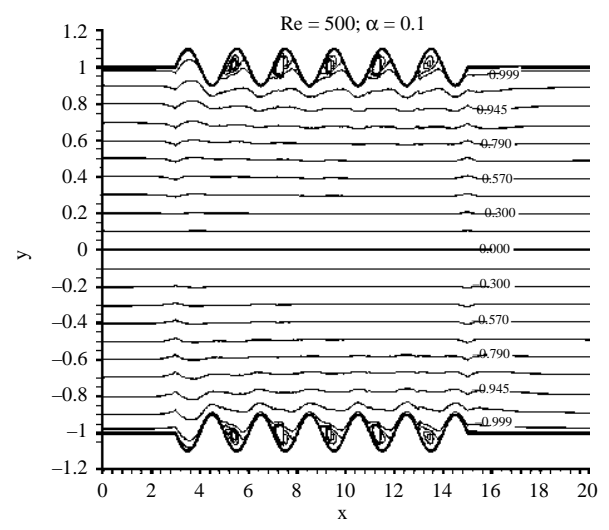

(a)

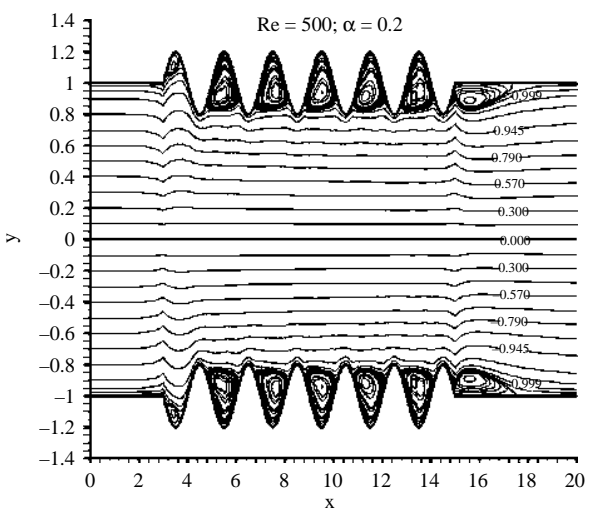

(b)

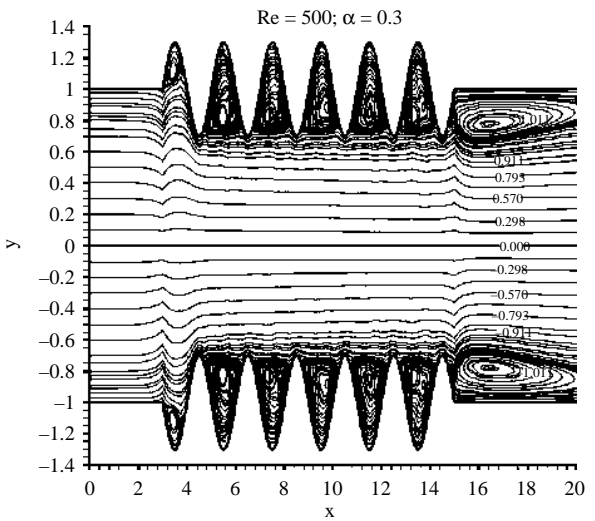

(c)

Notes: (a) $\alpha=0.1$; (b) $\alpha=0.2$; (c) $\alpha=0.3$ 


\section{Acknowledgements}

The authors would like to acknowledge the partial financial support provided by the Brazilian agencies and programs, FADESP and CNPq. One of the authors (R.M.C.) is also grateful for the kind hospitality of the School of Chemical Engineering and Laboratory of Solar Energy, during his technical visits to, respectively, the Universidade Federal do Pará and Universidade Federal da Paraíba, in 2007 and 2008.

\section{References}

Asako, Y., Nakamura, H. and Faghri, M. (1988), "Heat transfer and pressure drop characteristics in a corrugated duct with rounded corners", International Journal of Heat and Mass Transfer, Vol. 31, pp. 1237-45.

Balaras, E. (2004), "Modeling complex boundaries using an external force field on fixed Cartesian grids in large-eddy simulations", Computers \& Fluids, Vol. 33, pp. 375-404.

Cabal, A., Szumbarski, J. and Floryan, J.M. (2001), "Numerical simulation of flows over corrugated walls", Computers \& Fluids, Vol. 30, pp. 753-76.

Carvalho, T.M.B., Cotta, R.M. and Mikhailov, M.D. (1993), "Flow development in entrance region of ducts", Communications in Numerical Methods in Engineering, Vol. 9, pp. 503-9.

Castellões, F.V. and Cotta, R.M. (2008), "Heat transfer enhancement in smooth and corrugated microchannels, (invited lecture)", Proceedings of the 7th Minsk International Seminar on Heat Pipes, Heat Pumps, Refrigerators, Invited Lecture Minsk, Belarus, 8-11 September.

Chen, C.K. and Cho, C.C. (2007), "Electrokinetically-driven flow mixing in micro-channels with wavy surface", Journal of Colloid and Interface Science, Vol. 312, pp. 470-80.

Cotta, R.M. (1993), Integral Transforms in Computational Heat and Fluid Flow, CRC Press, Boca Raton, FL.

Cotta, R.M. (1994), "Benchmark results in computational heat and fluid flow: the integral transform method", International Journal of Heat and Mass Transfer, Vol. 16, pp. 381-93 (Invited Paper).

Cotta, R.M. (1998), The Integral Transform Method in Thermal and Fluid Sciences and Engineering, Begell House, New York, NY.

Cotta, R.M. and Mikhailov, M.D. (1997), Heat Conduction: Lumped Analysis, Integral Transforms, Symbolic Computation, Wiley, Chichester.

Cotta, R.M. and Mikhailov, M.D. (2006), "Hybrid methods and symbolic computations", in Minkowycz, W.J., Sparrow, E.M. and Murthy, J.Y. (Eds), Handbook of Numerical Heat Transfer, Wiley, New York, NY, pp. 493-552.

Cotta, R.M. and Pimentel, L.C.G. (1998), "Developing turbulent duct flow: hybrid solution via integral transforms and algebraic models", International Journal of Numerical Methods for Heat and Fluid Flow, Vol. 8, pp. 10-26.

Cotta, R.M., Santos, C.A.C., Quaresma, J.N.N. and Pérez Guerrero, J.S. (2005), "Hybrid integral transforms in convection-diffusion: recent applications in internal flow simulation (invited lecture)", Proceedings of the Fourth International Conference on Computational Heat and Mass Transfer, Paris-Cachan, France.

Dalal, A. and Das, M.K. (2007), "Numerical study of laminar natural convection in a complicated cavity heated from top with sinusoidal temperature and cooled from other sides", Computers \& Fluids, Vol. 36, pp. 680-700.

Figueira da Silva, E. and Cotta, R.M. (1996), "Benchmark results for internal forced convection through integral transformation”, International Communications in Heat and Mass Transfer, Vol. 23, pp. 1019-29.

\section{Integral transforms \\ solution}


$\mathrm{HFF}$

21,2

242
Figueira da Silva, E. and Cotta, R.M. (1998), "Mixed convection within vertical parallel-plates: hybrid solution by integral transforms", Numerical Heat Transfer Part A: Applications, Vol. 33, pp. 85-106.

Goldstein, L. and Sparrow, E.M. (1977), "Heat/mass characteristics for flow in a corrugated wall channel", Journal of Heat Transfer, Vol. 99, pp. 187-95.

IMSL Library (1991), MATH/LIB, IMSL Library, Houston, TX.

Kays, W.M. and London, A.L. (1984), Compact Heat Exchangers, McGraw-Hill, New York, NY.

Lima, G.G.C., Santos, C.A.C., Haag, A. and Cotta, R.M. (2007), "Integral transform solution of internal flow problems based on Navier-Stokes equations and primitive variables formulation", International Journal for Numerical Methods in Engineering, Vol. 69, pp. 544-61.

Lima, J.A., Pérez Guerrero, J.S. and Cotta, R.M. (1997), "Hybrid solution of the averaged Navier-Stokes equations for turbulent flow", Computational Mechanics, Vol. 19, pp. 297-307.

Machado, H.A. and Cotta, R.M. (1995), "Integral transform method for boundary layer equations in simultaneous heat and fluid flow problems", International Journal of Numerical Methods for Heat and Fluid Flow, Vol. 5, pp. 225-37.

Machado, H.A. and Cotta, R.M. (1999), "Analysis of internal convection with variable physical properties via integral transformation", Numerical Heat Transfer Part A: Applications, Vol. 36, pp. 699-724.

Marchioli, C., Armenio, V. and Soldati, A. (2007), "Simple and accurate scheme for fluid velocity interpolation for Eulerian-Lagrangian computation of dispersed flows in 3D curvilinear grids", Computers \& Fluids, Vol. 36, pp. 1187-98.

Naveira, C.P., Lachi, M., Cotta, R.M. and Padet, J. (2007), "Integral transform solution of transient forced convection in external flow", International Communications in Heat and Mass Transfer, Vol. 34, pp. 703-12.

Paz, S.P.A., Macêdo, E.N., Quaresma, J.N.N. and Cotta, R.M. (2007), "Eigenfunction expansion solution for boundary-layer equations in cylindrical coordinates: simultaneously developing flow in circular tubes", Numerical Heat Transfer Part A: Applications, Vol. 52, pp. 1123-49.

Pereira, L.M., Pérez Guerrero, J.S. and Cotta, R.M. (1998), "Integral transformation of the Navier-Stokes equations in cylindrical geometry", Computational Mechanics, Vol. 21, pp. 60-70.

Pérez Guerrero, J.S. and Cotta, R.M. (1992), "Integral transform solution for the lid-driven cavity flow problem in streamfunction-only formulation", International Journal for Numerical Methods in Fluids, Vol. 15, pp. 399-409.

Pérez Guerrero, J.S. and Cotta, R.M. (1995), "Integral transform solution of developing laminar duct flow in Navier-Stokes formulation", International Journal for Numerical Methods in Fluids, Vol. 20, pp. 1203-13.

Pérez Guerrero, J.S. and Cotta, R.M. (1996), "Benchmark integral transform results for flow over a backward-facing step", Computers \& Fluids, Vol. 25, pp. 527-40.

Pérez Guerrero, J.S., Quaresma, J.N.N. and Cotta, R.M. (2000), "Simulation of laminar flow inside ducts of irregular geometry using integral transforms", Computational Mechanics, Vol. 25, pp. 413-20.

Quaresma, J.N.N. and Cotta, R.M. (1997), "Integral transform method for the Navier-Stokes equations in steady three-dimensional flow", Proceedings of the Tenth International Symposium on Transport Phenomena, Kyoto, Japan, November-December. 
Santos, C.A.C., Quaresma, J.N.N. and Lima, J.A. (2001), Benchmark Results for Convective Heat Transfer in Ducts: The Integral Transform Approach, Editora E-Papers, Rio de Janeiro.

Silva, R.L., Santos, C.A.C., Quaresma, J.N.N. and Cotta, R.M. (2007), "Hybrid solution for developing laminar flow in wavy-wall channels via integral transforms", Proceedings of IMECE2007, ASME International Mechanical Engineering Congress \& Exposition, Paper No. IMECE2007-42965, Seattle, WA, USA, November, pp. 11-15.

Sunden, B. and Trollheden, S. (1989), "Periodic laminar flow and heat transfer in a corrugated two-dimensional channel", International Communications in Heat and Mass Transfer, Vol. 16, pp. 215-25.

Vasudevaiah, M. and Balamurugan, K. (2001), "Heat transfer of rarefied gases in a corrugated micro-channel”, International Journal of Thermal Sciences, Vol. 40, pp. 454-68.

Wang, C.C. and Chen, C.K. (2002), "Forced convection in a wavy-wall channel", International Journal of Heat and Mass Transfer, Vol. 45, pp. 2587-95.

Wolfram, S. (1999), The Mathematica Book, 4th ed., Wolfram Media, Cambridge.

Xiao, Q., Xin, R.C. and Tao, W.Q. (1989), "Analysis of fully developed laminar flow and heat transfer in asymmetric wavy channels", International Communications in Heat and Mass Transfer, Vol. 16, pp. 227-36.

\section{Corresponding author}

João N.N. Quaresma can be contacted at: quaresma@ufpa.br

To purchase reprints of this article please e-mail: reprints@emeraldinsight.com Or visit our web site for further details: www.emeraldinsight.com/reprints 